\title{
Mining Reviews for Product Comparison and Recommendation
}

\author{
Jianshu Sun, Chong Long, Xiaoyan Zhu, and Minlie Huang
}

\begin{abstract}
Recently, as the amount of customer reviews grows rapidly on product service websites, it costs customers much time to select and compare their favorite products. Researchers have been aware of this problem and many studies are investigated to mine the opinions from the online reviews. Unfortunately, few previous works give comparisons or recommendations among the products. In this paper, we propose an automated system to address this problem. We first build a product feature sentiment database from the reviews. Then we perform the comparison among various products from both subjective and objective perspectives on the feature level. Finally, product recommendations can be suggested according to the previous comparisons and an evolution tree constructed from the reviews. Experiment results demonstrate the effectiveness of the proposed approach in mining the digital camera reviews. And now a demo system is put in to practical use.
\end{abstract}

Index Terms-Review mining, comparison, recommendation, evolution tree.

\section{INTRODUCTION}

D UE to the emergence and development of Web2.0, more and more online review websites, such as Amazon [15] and Epinions [16], emphasize participation of the users. They encourage people to express their opinions on the products that they have purchased. These reviews are useful for both customers and manufacturers. However, it costs people a lot of time to find or collect useful information they want from so many reviews. Moreover, the judgment might be biased if only few reviews are analyzed. Instead of giving the users abundant but tedious reviews, it is better to summarize the reviews first, then perform comparisons among various products, and recommend good products according to the customer's demands.

Many researchers have proposed various approaches to mine product reviews. Hu et al. [1] and Liu et al. [2] developed a feature-based summarization approach on a large number of reviews of a product. In their work, they firstly tried to mine product features, and then identified opinion sentences with a positive or negative sentiment, which were summarized finally. In [6], the author proposed a novel relaxation-labeling technique to determine the semantic

Manuscript received November 5, 2008. Manuscript accepted for publication February 19, 2009.

Authors are with State Key Laboratory of Intelligent Technology and Systems Tsinghua National Laboratory for Information Science and Technology Department of Computer Science and Technology, Tsinghua University, Beijing 100084, China. (e-mail: bigtree2005@gmail.com, longc05@mails.tsinghua.edu.cn, \{zxy-dcs, aihuang\}@tsinghua.edu.cn). orientation of potential opinion words in the context of the extracted product features and specific review sentences. However, most of the foregoing work focuses on determining the sentiment polarity of a sentence or a review. Some researchers have noticed this limitation and try to evaluate the product by giving a sentiment score. In Scaffidi et al.'s work [8], they identified the product features and scores each product on each feature.

In this paper, we propose a system ${ }^{1}$ to compare various products, perform recommendations to the customers and visualize the results. People can compare the products on feature level to help them make informed decision. Moreover, the user can clearly tell the strengths and weakness of each product via comparison, as Fig. 1 shows. To recommend products, we build a visualized evolution tree to help customers find candidate products, such as the one with better performance but lower price in the same generation, or one of best-selling products in the next generation.

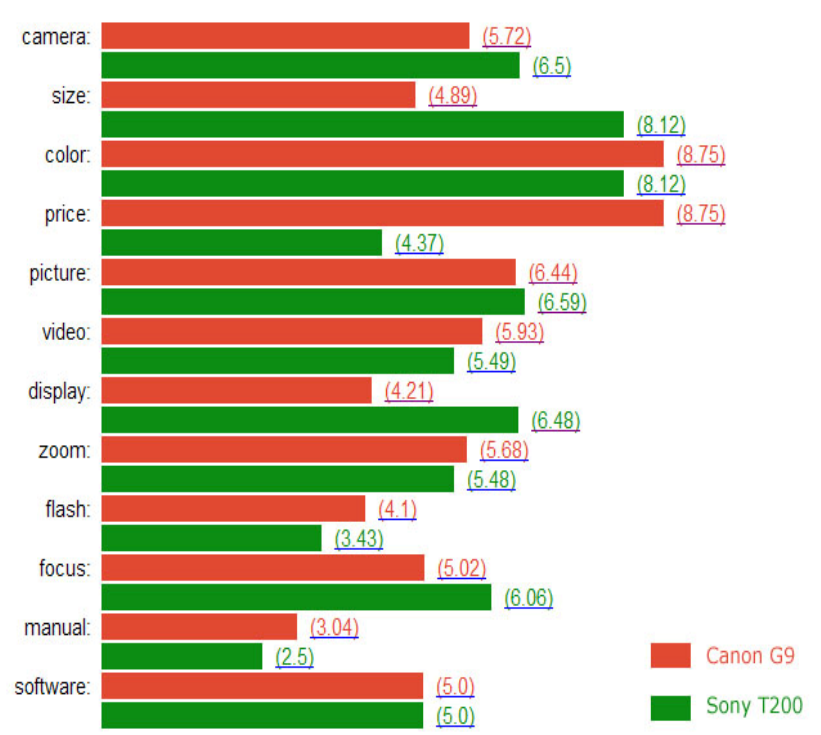

Fig. 1. Comparison visualization.

In summary, this paper has the following contributions:

- The proposed system can not only perform comparisons by mining reviews from the subjective perspective, but also incorporate product technical details to improve the comparison results from the objective perspective, which brings customers complete information.

\footnotetext{
${ }^{1}$ Please visit our online system at "http://60.195.250.72/procar/”
} 
- In our system, a new recommendation technique base on opinion comparison is proposed to suggest people some products with better performance. Moreover, we take the generation of product into consideration to ensure that the recommended products always have better physical performance.

- To the best of our knowledge, our system is the first one to construct the evolution tree of products. The evolution tree visualizes evolutionary process of products, which can indirectly recommend people potential favorable products.

The reminder of this paper is organized as follows: Section 2 describes some related work. The system architecture is discussed in Section 3. In Section 4, the proposed procedure is presented and the method is discussed. Experimental results are provided to confirm the effectiveness of the proposed approach in Section 5. Finally, the conclusion and future work are presented in Section 6.

\section{RELATED WORK}

\section{A. Comparative Opinion Mining}

Comparison is one of the most convincing ways of evaluation. For example, "The display of Sony T200 is good" provides different information against "The display of Sony T200 is better than Canon G9". Clearly, the latter provides more useful message about the camera Sony T200. Moreover, in many cases, customers want to compare products in a fine granularity, such as display of a digital camera or the battery life of a mobile phone. Before purchasing a product, a customer may compare various features in details among his/her candidates to make decisions. In this sense, product comparisons are essential in E-commerce.

Researchers have paid their attention to this aspect via various approaches. Liu et al. compares one product with another one by identifying comparative sentences [3] and mining relations between two entities with respect to some common features [4]. His methods can achieve a relatively high precision. However, for the comparative sentences are rare in product reviews, it is hard to perform comparisons among any products on any features. Liu et al's another work is implementing a prototype system called "Opinion Observer" [1] which focuses on analyzing and comparing opinions on the web. The system visualizes the comparison results so that the user is able to clearly see the strengths and weakness of each product in terms of various product features. However, the strength is simply generated by counting the number of positive opinions and negative opinions on one feature. In fact, the sentiment strength of each opinion is also very important when customers express their experience of a product. For example, the sentence "The display of Sony T200 is very excellent" obviously contributes more positive strength on the "display" feature than the ordinary statement "The display of Sony T200 is good". Pang et al [7] has focused on identifying opinion strength by classifying author's reviews into multi-point scale (e.g., one to five "stars"). While he cannot tell detailed scores on each feature, his work is just focusing on the document level.

Our work has gone further: we not only consider the strength of each customer's opinion, but also give a whole evaluation of each feature for a product, including incorporating product technical details. Comparison results based on each feature's evaluation have achieved a high precision.

\section{B. Product Recommendation}

Nowadays, recommendation is very common in electronic commerce's websites such as Amazon [15], Cnet [17]. When viewing a product's detailed description, customers are presented a product list similar as "What do customers ultimately buy after viewing this item?" or "Similar products". This recommendation technique mainly base on customer's visit records and previous classified categories. However, recommendation has much more requirements beyond that, including presenting products with better user experience and with suitable physical details.

In Scaffidi et al's work [8], they implemented a prototype system called Red Opal to score each product on each feature for the users to locate products rapidly based on features. But simply ranking products according to user specific desired feature cannot satisfy the customer's demand, such as "Please recommend some digital cameras whose screen, size and picture quality are better than those of Sony T200", and failed to consider the product generation. There are also some researchers who perform product recommendation by modeling user preferences to implement personalized recommendation. Zhang et al. [9] have proposed a contentbased personalized recommendation system which can learn user specific profiles from user feedback so that it can deliver information tailored to each individual user's interest. Differing from these personalized recommendation systems, our system focuses on statistical user opinions and recommends customers products with better subjective user experiences.

\section{SYSTEM ARCHITECTURE}

\section{A. Definitions}

Feature: A feature is an attribute/component of the product that has been commented on in reviews.

Opinion: The opinion of a feature in reviews is phrase (consecutive words) that expresses an opinion on the feature.

Feature-opinion pair: When a feature and its opinion occur in one sentence, we called them a feature-opinion pair.

For example, "photos" as a feature and "very good" as its opinion constitute a feature-opinion pair which expresses a positive opinion on the photo.

The photos come out very good.

Sentiment value (strength): A sentiment value is a scaled score from 0 to 1 , evaluating the positivity of a sentiment. While 1 represents the most positive sentiment, 0 represents the most negative sentiment. Neural sentiment is scored 0.5. 
Generation: Generation is a key indicator when measuring the development of products. Generation can be defined by various standards, such as selling periods, or primary features.

For example, Sony $T 100$ with selling period of "Feb.2007 Oct.2007" is next generation of Sony T1 with "Feb.2004 Feb.2005".

Another example, the generation development in memory card is from "SDRAM" to "DDR", to "DDR2", to current "DDR3".

Model number: A model number is an exactly representable value of a real type, usually referring to a series of numbers and letters. For example, "G9" is the model of product "Canon PowerShot G9 12.1MP Digital Camera with $6 x$ Optical Image Stabilized Zoom”.

Evolution tree: An evolution tree is a tree showing the evolutionary relationships among various products that are of the same series or brand. Please refer Fig. 7 in Section 5.C for a visualized demonstration.

Parent-child relation: Two products in one series, in which one product is directly the other one's next generation, constitute a parent-child relation.

For example, Canon G7 and Canon G9 constitute a parentchild relation.

\section{B. System Description}

As we can see in Fig. 2, the system firstly preprocesses the reviews from review websites, and then builds databases containing the evaluation results of product feature sentiments. Finally, the system provides visualized online services.

\section{Preprocessing:}

Firstly, we select some web pages containing reviews of the products that users are interested in. Then Struct Review Parser is employed to extract review information. Finally, after being processed by Sentence Splitter [14] and Stanford Parser [13], structured review datasets is prepared.

2. Database Construction/Building:

Feature opinion pairs mining and product feature sentiment evaluation are the two main steps when building Product Feature Sentiment Database. In the step of feature sentiment evaluation, SentiWordnet [10] is incorporated to determine the opinion sentiment strength, and Weighed Voting Method is employed to summarize all the opinions on the feature. After that, all the features of products are evaluated by scaled sentiment scores, which are finally stored into the database.

3. Online Services:

Three achievements of the system are exhibited by means of online web services:

a) Product Comparison.

Given two products and several features customers want to compare on, the system performs comparisons based on the scaled sentiment value from previous product feature sentiment databases, and provides a visualized comparison results, as
Fig. 1 shows. Detailed approaches will be discussed in Section 4.B.

b)Product Recommendation:

Given a current product and several features customers may care about, the system will list some recommended products, whose sentiment value on these features are higher than current product's, by previous comparison module. These candidate products are believed to have a better performance on these features.

c) Evolution Tree:

The evolution tree is generated by full information about products, consisting of reviews, brands, models and some primary technical details. While review's time distributions and some optional technical details decide the generation of a product, the brand and the model produce the parent-child relations in the tree.

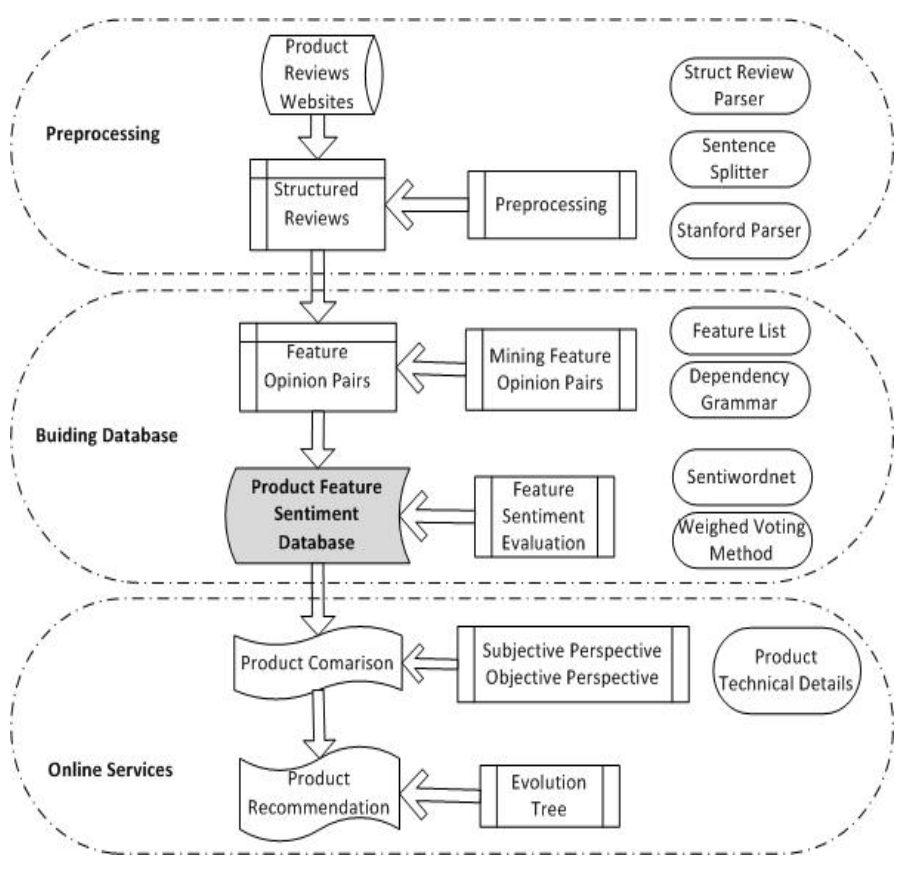

Fig. 2. System architecture.

\section{METHOD}

\section{A. Building Product Feature Sentiment Database}

\section{1) Mining feature opinion pairs}

Firstly, we mine feature $n$-grams list according to $\mathrm{Hu}$ et al.'s work [2]. Then we can retrieve feature-opinion pairs from reviews using these feature n-grams. As Fig. 3 shows, once we locate the feature word in a sentence, we can find a dependency path [12] "photos (NNS) - come (VBN) - good $(\mathrm{JJ})$ - very (RB) " in which "photos" and "very good" can be labeled as feature and opinion respectively. In our system, we view opinion as "adjective n-grams" more than a word from a fixed opinion keyword set compared to Zhuang et al.'s work [5]. 
TABLE I

FOUR CATEGORIES BY INTENSE FACTORS

\begin{tabular}{llll}
\hline \hline category & adverbs & intense factor & adjective n-grams (sentiment value) \\
\hline intense words & very, too, -est & 1.2 & very excellent $(0.75=0.625 * 1.2)$ \\
ordinary words & relatively & 1 & relatively excellent $(0.625)$ \\
weak words & just, & 0.8 & just excellent $(0.5=0.625 * 0.8)$ \\
negative words. & not, seldomly & $\frac{1 \text {-sentiment }}{\text { sentiment }}(*)$ & not excellent \\
& & $(0.375=0.625 *(1-0.625) / 0.625)$ \\
\hline
\end{tabular}

Comments for Table I: The sentiment value of original "excellent” is 0.625 . (*) The sentiment value of negative adjective n-grams equals the result subtracting the "sentiment" of original adjective from 1 . The intense factor in the table is filled for consistency.

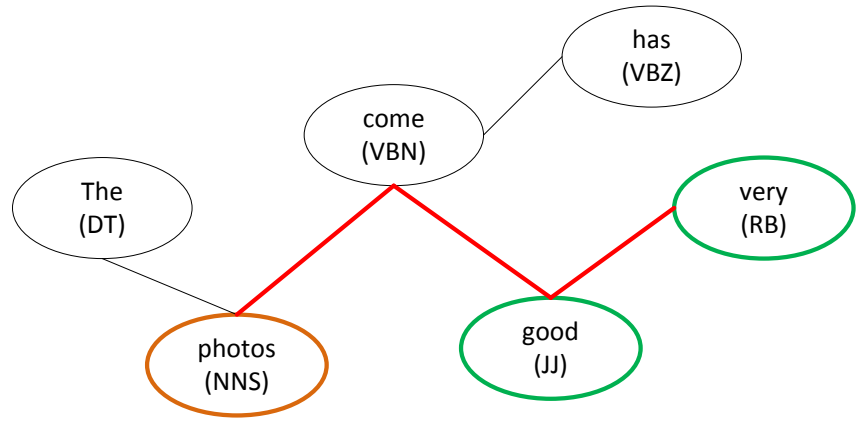

Fig. 3. Dependency grammar graph. This shows the dependency grammar graph generated by Stanford Parser [13]. The broad line indicates the dependency path from feature ("photos") to opinion ("very good").

\section{2) Evaluate product feature sentiment}

Since we want to compare products on a specific feature, product feature sentiment evaluation is essential in our work.

After finishing feature-opinion pairs mining procedure, the key problem now is how to assess the opinion on product features, which contains two sub-problems: how to evaluate one feature-opinion pair's sentiment and how to summarize all sentiment values on one product feature. Fortunately for the first problem, SentiWordnet [10] provides a list of words, in which each one has a positivity-score and a negativity-score with a scope of $[0,1]$. We expand the word list a newly ngrams list, as we called "Expanded SentiWordnet", each word of which has a scaled sentiment value. The expanding rules mainly deal with "adjective n-grams" by multiplying the sentiment value of the adjective word by an intense factor $a$ when there is an adverb before the adjective word. We previously classified the adverbs in SentiWordnet into four categories: intense words, ordinary words, weak words and negative words. Examples of four kinds of adverbs are showed as Table I.

We develop a weighted voting method to deal with the second problem. The method combines the opinion n-grams' frequency and its own sentiment value. Formally, for a product $i$, the score feature _score on a feature $j$ can be calculated by Equation 1 .

$$
\begin{aligned}
& \text { feature_score }(i, j)= \\
& \sum_{\text {opinion_weight }}>=2 \text { opinion_weight } t_{k} \times \text { opinion }_{-} \text {score }_{k} \text {. }
\end{aligned}
$$

In Equation 1, the opinion n-grams frequency opinion _ weight is calculated by all the feature-opinion pairs related the feature. An opinion_score is looked up from the "Expanded SentiWordnet". In order to remove the noise, we only consider the opinion n-grams that occur more than once.

For each product, we can extract all the feature-opinion pairs from all the reviews of the product, and integrate all the sentiments of pairs into the feature level. After the features of all the products are evaluated in the same scale, we can store the results into databases.

\section{B. Product Comparison and Recommendation}

Differing from a 5-star schema in Amazon [15], our system compares products on the feature level. For example, from statistical results on Amazon, Canon G9 and Sony T200 have a 4.3-rating and 3.74-rating respectively, which means Canon G9 has better overall user experience than Sony T200. But in our system, we want to tell that on "display" feature, Sony T200 is better than Canon G9 (See the Fig. 4 for visualized results).

The system performs product comparisons based on previous product feature sentiment database. Formally, for any two products $\mathrm{A}$ and $\mathrm{B}$, considering the feature $\mathrm{j}$, whether product $\mathrm{A}$ is better than product $\mathrm{B}$ depends upon the value of Equation 2.

feature_score $(A, j)>$ feature_score $(B, j)$ ?

Most of time, two products have clearly different sentiment scores on identical feature. However, what if two products have the same or similar sentiment scores on the same feature? Here is an example: the "picture", "zoom" feature of "Sony T200", "Canon G9", "Canon SD750" display in the left chart of Fig. 4. The three products have achieved similar scores. An explanation of this phenomenon is that the three products have so high picture pixels (at least 7.1MP) that over satisfy ordinary people's demands, leading people unable to distinguish them. In addition, there are some circumstances when people may have biased opinions or have no comments on one feature. All of these demonstrate that subjective views have their own limitations. 
To deal with this problem, the system incorporates objective product technical details, which describe some product's own numerical properties, such as size, picture revolution, optical zoom, etc. After scaling the properties among all the products, the system adds these factors to final comparison measurements. Experiments show that the combination of subjective views and objective views make the system achieve effective improvements. Fig 4a presents traditional results, while Fig. 4b reflects a good result on "picture", "zoom" after adding product technical details.

When we already have product comparison results, we can start our recommendation procedure. Firstly, we select all the products as candidates that have the same generation with customer's current product. Secondly, product comparison is executed between each of the candidates and the current product base on the customer selected features, and the losers will be removed from the candidates. Finally, the candidates will be displayed as the recommended products, ranked by general feature scores. These recommended products are believed to have a better performance on the customer selected features.

\section{Evolution Tree}

An evolution tree describes the evolutionary process of one kind of product. Moreover, an evolution tree can actually recommend people potential favorable products under the visualization surface. With a simple glance of his/her current product in the evolution tree, a customer may begin to discover more suitable products. First, he/she may care about whether the product of next generation has come out so that he/she can compare the performances. Then, he/she may scan other ones in the same generation to seek the products with better performance but lower price. Still, he/she may compare these products with current one in details using our system so that he/she can find the most suitable one. In this sense, the evolution tree is a large visualization facilitate for customers selecting their suitable products.

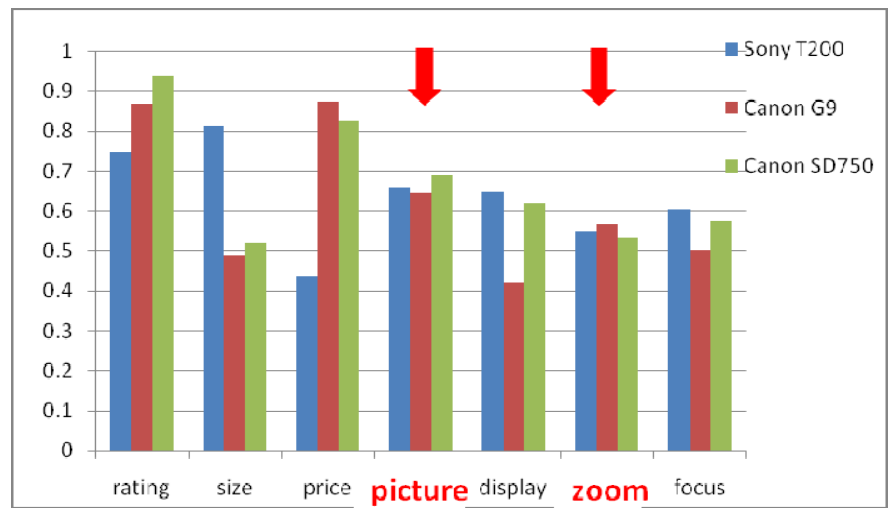

Fig. 4a. Comparison results of three digital cameras. The chart shows feature scores distributions from the subjective perspectives

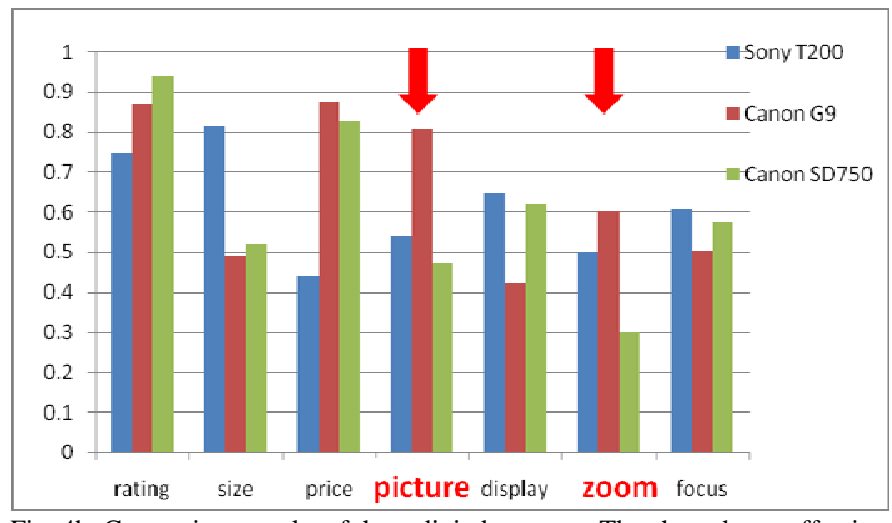

Fig. 4b. Comparison results of three digital cameras. The chart shows effective improvements after incorporating objective product technical details, especially on picture and zoom. The first feature "rating" is obtained from Amazon.

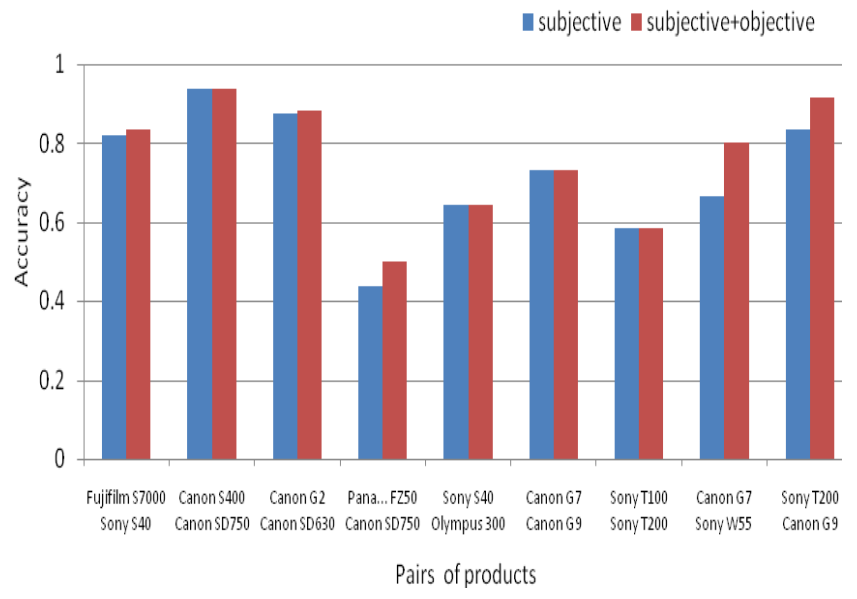

Fig. 5. Accuracy performance of comparison results over 9 randomly selected pairs of products. The subjective represents comparison results only from product feature sentiment database. The subjective+objective represents the results after incorporating the product technical details.

Now let's consider how to construct the evolution tree. In the system, we propose a novel multi-knowledge based constructing method, which involves review dates, brands, models and some primary technical details. The step-by-step approach is implemented as follows:

\section{1) Determining generations}

Generation can be defined by various standards. However, in our system, we measure the generation by both the selling periods and several primary features. We can obtain a time distribution from product's reviews, which is viewed as the main feature (different concept from product feature) of generation, with several core features (core features can be customized across products) as a plus. For example, for products of "digital camera", we divide "Feb $1^{\text {st }}$, 1999 Jan $31^{\text {st }}$, 2009" (containing all review times) by 3 months into 40 slots, and count the number of reviews falling into each slot. The 40 numbers constitute the main part of the feature vector. Besides, the number of mega picture resolution and optical zoom are also attached to the feature vector. A scaling operation will be performed in order to balance the 
contribution between review time distribution and core features.

After the scaled feature vector is sent to a cluster procedure, all the products are divided into several clusters. The number of clusters is predefined as N. In our experiments, we find $\mathrm{N}=3$ leads to the best visualization of the evolution tree. By comparing the average time of product reviews in each cluster, we label each cluster as "generation 0", "generation 1 ”, “generation 2”, etc.

\section{2) Building parent-child relations}

Parent-child relations are constructed mainly according to product brands and product models, which means product's full name is essential in this step. This is reasonable, because judging whether "Canon G9" is the next generation of "Canon G7" only depends on the product "full name" information [15].

From top to bottom, the products in the current generation try to find parents in the hyper-generation. If being found, the product and the parent will be connected by a parent-child relation. If not, the product will recursively try to find parents in the next hyper-generation.

To ensure each product can find a parent, we construct the tree root as "generation type" by the product type, such as "digital camera", "mobile phone”, followed by constructing node "generation -brand" by all the product brands, such "Canon", "Sony" (Please refer to Fig. 7 in Section 5.C for a visualized demonstration). Even if a product cannot find a real product parent, it can still find his brand node in the "Generation brand".

\section{3) Merging the same parents}

It is noticed that a child may be connected with more than one parent in the previous steps, so we need to merge all the parents that have the same child, which means the node of evolution tree may contains more than one product. This step is necessary for maintaining the tree structure. For example in Fig. 7, "Sony W50" is both the child of "Sony W1" and "Sony $W 5$ " in the hyper generation. So we merge the two parent nodes into one node as "Sony W Sony W5".

Note that steps 2 and 3 can be computed together. We present them separately for clarity. Please refer to Fig. 7 in Section 5.C for visualized evolution tree of a digital camera.

\section{EXPERIMENTAL RESULTS}

To evaluate our proposed method, we perform extensive experiments on a corpus consisting of 23,585 product reviews from Amazon [15]. There are total 209 types of digital cameras, each of which has more than 50 reviews.

\section{A. Product Comparison}

1) Mining feature opinion pairs

To test the performance of product comparison module, 9 pairs of products with 18 product features are randomly selected from the 209 products.

Three people from different backgrounds are invited to annotate the comparison results. Each comparison on each feature is labeled by three annotators independently, with one of the following three labels according to the comprehension of feature sentence and related reviews:

- Label ' $T$ ': the left product is better than the right product on the feature.

- Label ' $F$ ': the left product is worse than the right product on the feature.

- Label 'E': the two products have the same or similar sentiment score on the feature.

Three people's annotations are combined into the final annotation as follows: If more than one annotator has the same label, the label is the final annotation. If three annotators have different labels respectively, the final annotation is ' $E$ '.

From Fig. 5, we can see that our system have achieved encouraging performance on the product pairs. Our final average accuracy is 0.759 , which means that the system can correctly performs comparisons on 14 out of 18 features in average. And when focusing on each pairs, we can find out limited differences. This is reasonable because some pairs have similar performance but some ones differ apparently, reflected by not only their ratings in Amazon, but also product technical details. For example, in the second pair, "Canon S400" has a 3.56-rating, while "Canon SD750" has a high 4.70-rating. In the last pair, "Sony T200" and "Canon G9", famous for their pocket size and advanced performance respectively, have tremendous differences in product technical details. The third pair of "Panasonic FZ50" and "Canon SD750" has the same ratings in Amazon and similar physical performance, which leads a relative low accuracy of the comparison results.

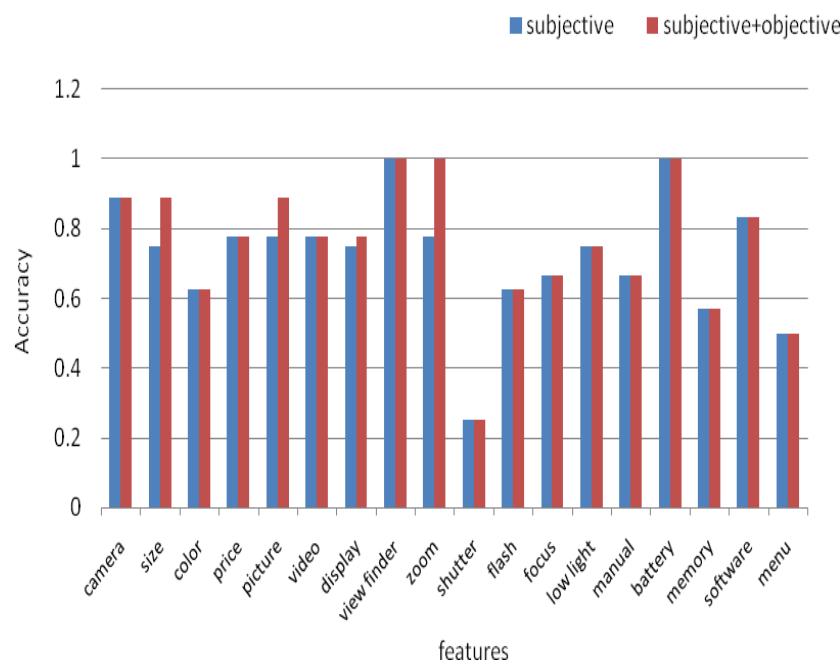

Fig. 6. Accuracy performance of comparison results over 18 product features.

From another perspective, Fig. 6 demonstrates the results over 18 product features. As you can see, the system achieves different accuracy performance over different features. On "view finder", "zoom" and "battery", the system even gives no errors. This is mainly because customers care about these features most when they choose a product. On the other side, common customers pay less attention to "shutter" or "menu", which leads to a bad performance on these features. An 
explanation for bad performance on some features such as "color" is due to different personal preferences.

We can see some improvements after incorporating the product technical details on the features of "size", "picture", "display" and "zoom", which proves our effective methodology of bringing objective parameters of product technical details to comparison measures from both Fig. 5 and Fig. 6.

\section{B. Product Recommendation}

In the experiment of product recommendation, 5 products and several features according to the annotator's selection are randomly chosen from total 209 products of digital camera. To test the performance of product comparison module, each recommended product in at most top10 (may be less than 10) need to be labeled ' $\mathrm{T}$ ' as good suggestion or ' $\mathrm{F}$ ' as bad suggestion, according to annotators' judgments.

Table II shows the average accuracy of top10 recommended products by three annotators. Recommendation tends to provide only candidates for customers to select. Therefore, the accuracy in Table II has already confirmed the system's effectiveness when performing recommendations.

TABLE II

ACCURACY PERFORMANCE OF RECOMMENDATION

\begin{tabular}{lll}
\hline \multicolumn{2}{c}{ ACCURACY PERFORMANCE OF RECOMMENDATION } \\
\hline \hline current product & features & $\begin{array}{l}\text { Accuracy of } \\
\text { Top10 }\end{array}$ \\
\hline Fujifilm S7000 & picture, battery, price & 0.67 \\
Canon SD1000 & price, picture & 0.5 \\
Sony T200 & display & 0.7 \\
Canon G9 & battery & 0.6 \\
\hline
\end{tabular}

\section{Evolution Tree}

It is difficult to evaluate the performance of evolution tree with a tree structure, because there are not any standard evolution trees so far.

However, we can still discuss the visualization information the evolution tree represents or check whether the evolution tree is reasonable. Because there are some basic rules a normal evolution tree cannot violate, such as parent-child relation cannot be reverse, a too old product cannot have the same generation with a latest product. Let's refer a typical generated tree for more discussions.

Fig. 7 describes an evolution tree of digital camera. Except for "generation type" and "generation brand" for maintaining the tree structure, there are four series of products consists of "Sony $T$ series", "Sony W series", "Canon G series" and "Canon SD series", which clearly shows the evolutionary process. For example, "Sony T1 - Sony T100 - Sony T200" as "Sony $T$ series", represents a kind of Sony card machine, with a pocket size, a big screen even touch screen and high picture quality. The partial order of father-son relation can be verified by the following knowledge in Table III.
TABLE III

THE EVOLUTIONARY PROCESS OF "SONY T SERIES".

\begin{tabular}{lllll}
\hline \hline products & model & $\begin{array}{l}\text { picture } \\
\text { resolution }\end{array}$ & zoom & $\begin{array}{l}\text { review time } \\
\text { distribution }\end{array}$ \\
\hline Sony $T 1$ & $T 1$ & $5 M P$ & $3 x$ & Feb.2004 Feb.2005 \\
$\begin{array}{l}\text { Sony } \\
\text { T100 }\end{array}$ & $T 100$ & $8.1 M P$ & $5 x$ & Feb.2007 Oct.2007 \\
$\begin{array}{l}\text { Sony } \\
\text { T200. }\end{array}$ & $T 200$ & $8.1 M P$ & $5 x$ & May.2007 now \\
\hline
\end{tabular}

On the other hand, let's check the products that locate in the same generation. Products in the bottom generation are all current-selling commodities a relative high performance, while products lying in the upper generation are recently outdated commodities. And the top generation is mostly the first products in their series, representing the first digital camera in the history.

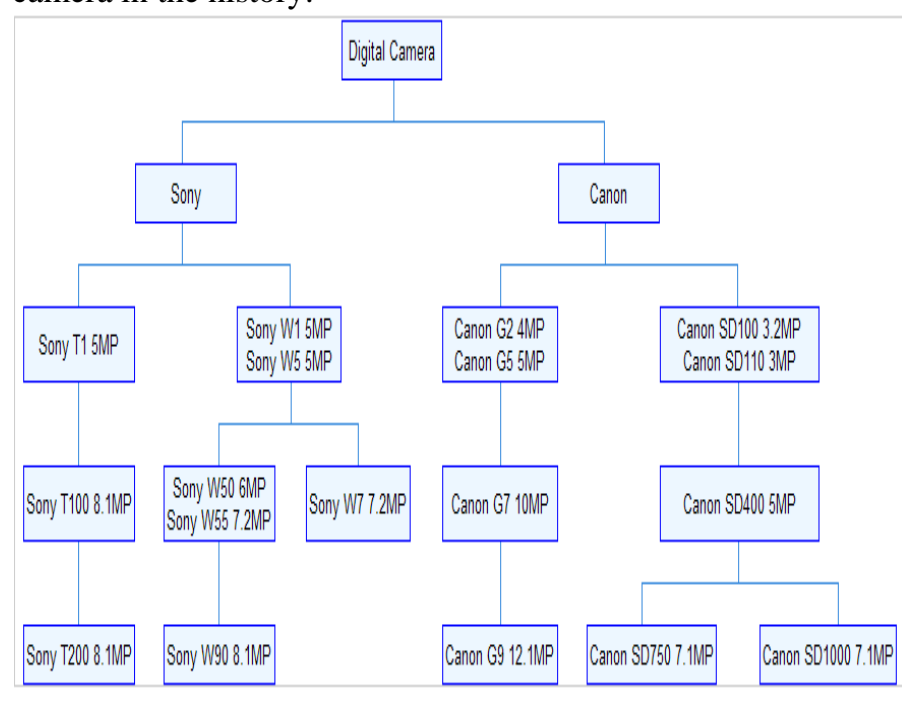

Fig. 7. An evolution tree generated by the system. This shows the evolutionary process of some digital cameras, in which root node "Digital Camera" is viewed as "generation type" and "Sony" node and "Canon" node are viewed as "generation brand" for visualization.

\section{CONCLUSION AND FUTURE WORK}

In this paper, we have proposed an automated system to compare and recommend products for customers from both subjective and objective perspectives. Moreover, we have developed a methodology to construct evolution tree of products, which not only provides a visualization of product evolutionary process, but also help customers seek potential better products. Experiment results show the effectiveness of the proposed approach. In the future work, we will consider extracting sentiment information from other types of opinion sources, and try to provide a summarized comparison passage of any two products.

\section{ACKNOWLEDGMENT}

The work was supported by NSFC under grant No.60621062 and 60803075, the National Basic Research Program (973 project in China) under grant 
No.2007CB311003, and Microsoft joint project "Opinion Summarization toward Opinion Search". The work was also supported by a grant from the International Development Research Center, Canada.

\section{REFERENCES}

[1] Bing Liu, Minqing Hu and Junsheng Cheng, "Opinion Observer: Analyzing and comparing opinions on the web," in Proceedings of WWW 2005, pp. 342-351, 2005.

[2] Minqing $\mathrm{Hu}$ and Bing Liu, "Mining and summarizing customer reviews," in Proceedings of ACM-KDD 2004, pp. 168-177, 2004.

[3] Nitin Jindal and Bing Liu, "Identifying comparative sentences in text documents," in Proc. of SIGIR-06, pp. 244-251, 2006.

[4] Nitin Jindal and Bing Liu, "Mining comparative sentences and relations," in Proc. of AAAI'06, pp. 244-251, 2006.

[5] Li Zhuang, Feng Jing, and Xiaoyan Zhu, "Movie review mining and summarization," in Proc. of CIKM, pp. 43-50, 2006.

[6] Ana-Maria Popescu and Oren Etzioni, "Extracting product features and opinions from review," in Proc. of EMNLP-05, pp. 339-346, 2005.

[7] Bo Pang and Lillian Lee, "Seeing stars: Exploiting class relationships for sentiment categorization with respect to rating scales," in Proc. of ACL 2005, pp. 115-124, 2005.

[8] Christopher Scaffidi, Kevin Bierhoff, Eric Chang, Mikhael Felker, Herman Ng and Chun Jin, "Red Opal: Product-Feature Scoring from Reviews,” in Proc. of ACM EC, pp. 182-191, 2007.

[9] Yi Zhang and Jonathan Koren, "Efficient Bayesian hierarchical user modeling for recommendation systems," in Proc. of SIGIR-07, pp. 4754, 2007.

[10] Andrea Esuli and Fabrizio Sebastiani, "Sentiwordnet: A publicly available lexical resource for opinion mining," in Proceedings of LREC 2006, pp. 417-422, 2006.

[11] Yiming Yang and Jan O. Pedersen, "A comparative study on feature selection in text categorization," in Proc. of International Conference on Machine Learning (ICML), pp. 412-420, 1997.

[12] Marie-Catherine de Marneffe, Bill MacCartney and Christopher D. Manning, "Generating typed dependency parses from phrase structure parses," in Proc. of LREC 2006, 2006.

[13] Stanford Parser, http://www-nlp.stanford.edu/software/lex-parser.shtml

[14] SentParBreaker, http://text0.mib.man.ac.uk:8080/scottpiao/sent_detector

[15] Amazon, http://www.amazon.com

[16] Epinions, http://www.epinions.com

[17] Cnet, http://www.cnet.com 